\title{
Heuristic and supervised approaches to handwritten annotation extraction for musical score images
}

\author{
Eamonn Bell · Laurent Pugin
}

Received: date / Accepted: date

\begin{abstract}
Performers' copies of musical scores are typically rich in handwritten annotations, which capture historical and institutional performance practices. The development of rich, interactive interfaces to explore digital archives of these scores and the systematic investigation of their meaning and function will be facilitated by the automatic extraction of handwritten score annotations. We present several approaches to the extraction of handwritten annotations of arbitrary content from digitized images of musical scores. First, we show promising results in certain contexts when using simple unsupervised clustering techniques to identify handwritten annotations in conductors' scores. Next, we compare annotated scores to unannotated copies and use a printed sheet music comparison tool, Aruspix, to recover handwritten annotations as additions to the clean copy. Using both of these techniques in a combined annotation pipeline qualitatively improves the recovery of handwritten annotations.

Recent work has shown the effectiveness of reframing classical optical musical recognition tasks as supervised machine learning classification tasks. In the same spirit, we pose the problem of handwritten annotation extraction as a supervised pixel classification task, where the feature space for the learning task is derived from the intensities of neighboring pixels. After an initial investment of time required to develop dependable training data, this approach can reli-
\end{abstract}

Eamonn Bell

Department of Music

Columbia University

New York, NY 10025

United States of America

E-mail: epb2125@columbia.edu

Laurent Pugin

RISM (Switzerland)

Hallwylstrasse 15

CH-3000 Bern 6

Switzerland

E-mail: laurent.pugin@ rism-ch.org ably extract annotations for entire volumes of score images without further supervision. These techniques are demonstrated using a sample of orchestral scores annotated by professional conductors of the New York Philharmonic Orchestra. Handwritten annotation extraction in musical scores has applications to the systematic investigation of score annotation practices by performers, annotator attribution, and to the interactive presentation of annotated scores, which we briefly discuss.

\section{Introduction}

Handwritten annotations enrich documents with commentary and editorial revisions. Performers' annotations of musical scores indicate their musical preferences, and even authorial revisions when a performer is also the composer or editor of a score. To date, there has been little emphasis on the identification and extraction of annotations from musical scores. A set-up typical of the document analysis literature is the identification and separation of regions of handwritten text from scanned typewritten documents.

Early attempts at handwriting detection in scanned documents exploited the structural differences between handwritten and machine-printed characters to differentiate handwritten text annotations from printed text [13, 4]. Character-level probabilistic models have been used by [6] in the same task, while [5] achieved word-level annotation identification using Gabor filters.

Most of the techniques described above exploit text-specific features of handwriting and therefore cannot be used to extract non-text annotations. These kinds of annotations are typical on musical scores and include phrasing slurs, crescendo and decrescendo markings, cues, and countless miscellaneous annotations of value to the performer both in rehearsal and in performance. More general approaches show good re- 
sults, extracting both handwritten and other basic geometric annotations using a probabilistic graphical model that does not rely on text-specific structural differences between handwriting and printed matter $[11,14]$.

In this paper, we first describe three heuristic annotation extraction techniques that are useful when unmarked copies of scores are available, and/or when the annotations are marked in a color that is distinct from the underlying paper and printed matter.

Many archival annotated scores have been digitized in full color. Therefore, information about the distribution of pixel intensities in digital score images can be used as the input to classical image segmentation algorithms. We use this information to perform color separation by quantizing the color space of the annotated score images with the use of the unsupervised, k-means clustering algorithm.

Furthermore, since most printed music scores of interest have been published at scale, we can often find a clean version of the score against which to employ image-comparison methods to perform annotation extraction. For instance, the IMSLP/Petrucci Music Library Project provides thousands of clean copies of public-domain scores for download. Our approach follows [9], using local arrangements of feature points to align both clean and annotated versions of a document in order to extract annotations.

The Aruspix toolkit [12] enables the comparison of different copies of the same edition of printed music by image superimposition. Image processing is applied to de-skew, rotate, and resize the score images in order to align them. The differences between the two copies can be then extracted into a separate image array or highlighted in a graphical user interface. We show how both color separation and comparisonbased methods can be used to achieve score annotation exraction, first individually, and then together in a novel joint application that qualitatively improves annotation detection.

We then change tack and use this heuristic image processing pipeline to prepare a test dataset of full-color annotated score images, where pixel-level binary class labels indicate whether a pixel does or does not form part of a handwritten annotation. A comparatively small sample of ground truth can be used to train familiar pixel-level supervised classification algorithms, which can predict the location of score annotations without the subsequent need for color quantization or comparison with an unmarked copy.

In this paper we aim to motivate the development of a more extensive and representative dataset of class-labeled annotated score images, that goes beyond the test data we describe below. We suggest that our heuristic annotation extraction techniques assist in the rapid curation of such a ground truth, and that our demonstration of the potential of pixellevel supervised classification techniques shows that this endeavor is worthwhile.

\section{Heuristic annotation extraction}

\subsection{Color separation using k-means clustering}

It is common for conductors to use colored pencils in the preparation of scores for performance. As a consequence, many handwritten annotations to conductors' scores are often in a color not represented in the printed sheet music. We used color quantization to identify regions of an image array that correspond to colored annotations. Figure 1 summarizes this approach (Pipeline 1). A common method for computing a quantized color map from a source image (1) is to use an unsupervised clustering algorithm on the values of image pixels. k-means clustering is an unsupervised classification algorithm that learns to assign a finite number of class labels to observations. We used $\mathrm{k}$-means clustering on a subsample of the marked score to train a classifier that partitions pixels according to their position in the Lab perceptual color space into a number of visually similar clusters (2). The user identifies the clusters corresponding to the annotation colors, and the pixels corresponding to these clusters are selected and included in an image array that contains the annotated regions of the score image (3).

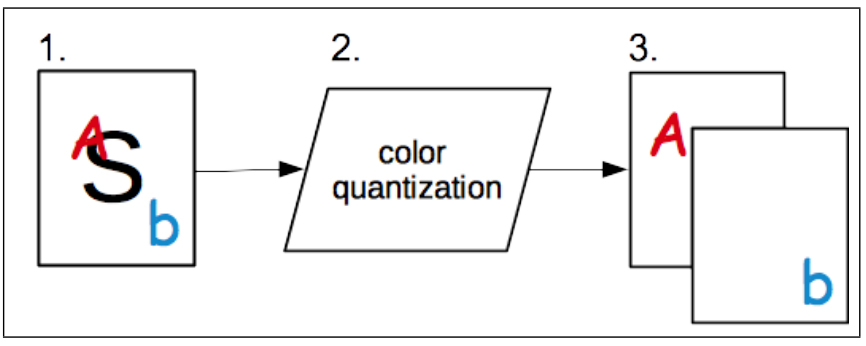

Fig. 1: Extraction of colored annotations using color quantization on annotated score images (Pipeline 1). Here, and in subsequent figures, the black $\mathrm{S}$ represents regions of printed matter on the score image, while the colored letters represent the annotated regions on that image.

One disadvantage of this approach is that the k-means clustering algorithm is not deterministic, requiring random initialization of proposed cluster centers. Certain initializations of k-means clustering return quantizations that will not assign all the pixels of a colored annotation to the same cluster. One solution to this problem that we develop below is to bias the data upon which the k-means classifier is trained so that it is more likely to contain the pixel color values of annotated regions of the image. This improves the likelihood that the algorithm will converge on clusters that are representative of annotated regions of the score image. This approach, however, has limited applicability to scores that have been annotated using a pen or a pencil close in color 
to the color of printed ink on paper, or to grayscale scans of annotated scores.

\subsection{Comparing marked and unmarked score images}

To account for this eventuality, we used Aruspix, a printed score comparison toolkit, to compare marked scores images with unmarked versions of their corresponding printed editions.

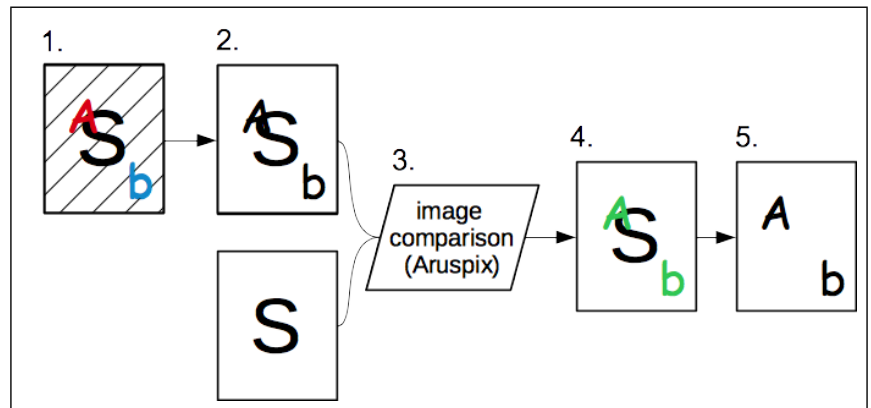

Fig. 2: Comparing marked and unmarked scores to generate an image "diff" containing annotated regions (Pipeline 2).

This annotation extraction process (Pipeline 2) is summarized in Figure 2. Original color scans of marked scores (1) are pre-processed before grayscale conversion to adjust for variations in the neutral color of the paper. TIFFs of the same page of sheet music (2) are passed to Aruspix (3) as input along with alignment markers determined visually by the user. Aruspix returns an image array that shows pixels that are judged to be additions to the unmarked score in green (4); these additions to the score are stored in a blackand-white image array (5).

Since Aruspix currently only supports grayscale TIFFs at all stages of its internal processing pipeline, this visual "'diff" does not preserve color information if annotations have been made in color. However, this technique is applicable for the extraction of scores that have been annotated using a non-colored pen or pencil, or indeed to grayscale scans of annotated scores, in contradistinction to Pipeline 1.

\subsection{Combined approach}

The two approaches described above can be combined in Pipeline 3, as summarized in Figure 3. We start with the output of the image comparison pipeline, a black-and-white image array containing annotated regions of the original image (1). This array is converted to a mask (2) which reveals the annotated regions and hides the unannotated regions of the score image. The mask is then morphologically transformed by dilation (3). This increases the area revealed by the mask beyond the regions returned by the image comparison step. This step also improves the connectivity of these regions. The mask is applied (4) to the original image, and the color values of revealed pixels are used to train the kmeans classifier that then performs color quantization (5). As before, clusters corresponding to annotation colors are filtered into separate image arrays.

This combined approach utilizes a mask defined by the results of the simple comparison pipeline to ensure that the input to the k-means clustering process contains the color values of as many pixels in annotated regions as possible. We aim to reduce the number of pixels representing blank paper or printed ink in the training data for the color quantization step. In turn, this increases the likelihood that the clustering algorithm will converge on clusters that represent the annotation colors in the original image, as opposed to elements of the printed score. The same mask may be reapplied at the end of the pipeline, obscuring any mislabeled regions of the whole score image that are not in the neighborhood of annotated regions determined by the comparison step.

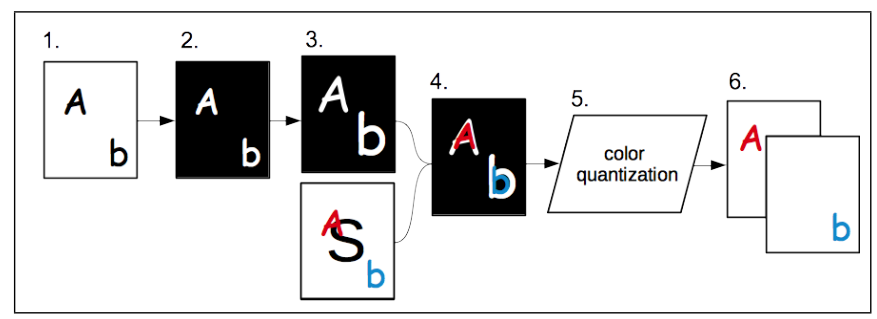

Fig. 3: Combined approach, using results from image comparison pipeline to bias color quantization algorithm to improve convergence on colors representing annotations (Pipeline 3).

\subsection{Evaluating heuristic extraction methods}

We show a cropped region of the result of applying the three processing pipelines to the example marked score in Figure $4 .{ }^{1}$ A post-processing filtering step was used to eliminate pixels that were unlikely to correspond to annotated regions. In order to eliminate artifacts resulting from the undesired extraction of vestigial remains of vertical and horizontal score elements and isolated single-pixel anomalies, three successive median filters with different window geometries were applied after each pipline was completed.

Figure $4 \mathrm{~b}$ shows the results of extraction by color separation, using k-means clustering $(k=7)$ in Lab perceptual color space (Pipeline 1). The unsupervised quantizing

1 The Python code used to implement each of these pipelines is available from the corresponding author (Bell), on request. 


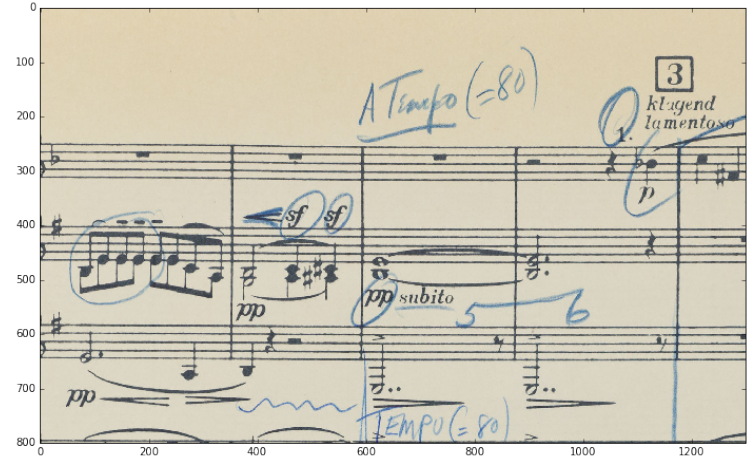

(a) Original score image (from Mahler, Kindertotenlieder (marked by Leonard Bernstein).

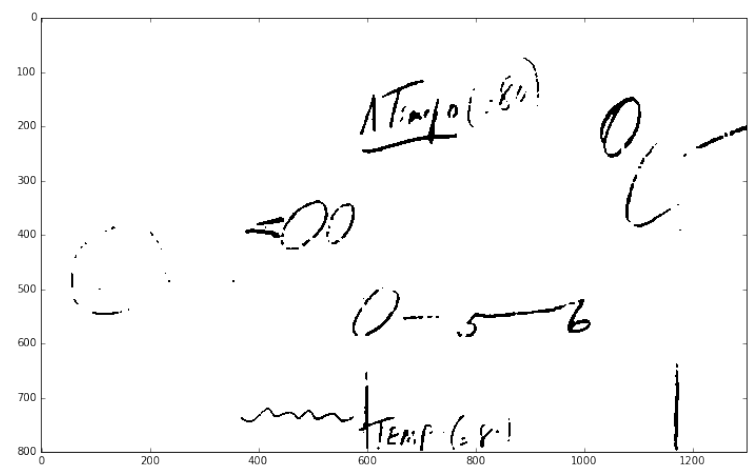

(b) Results of color separation (Pipeline 1).

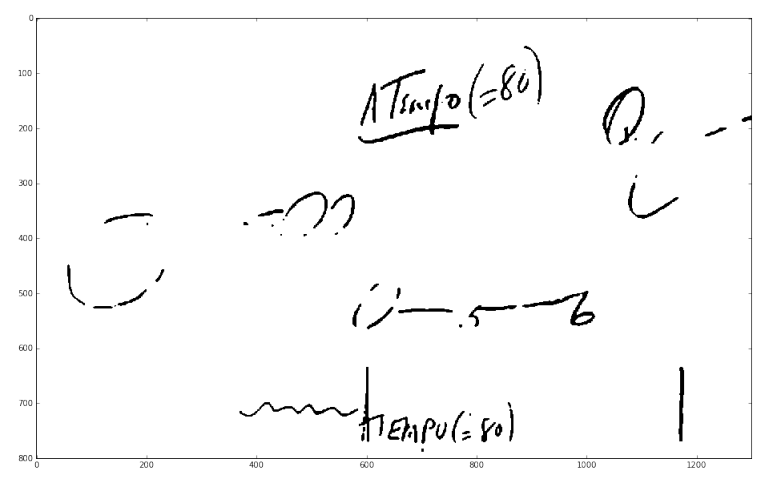

(c) Results of image comparison using Aruspix (Pipeline 2).

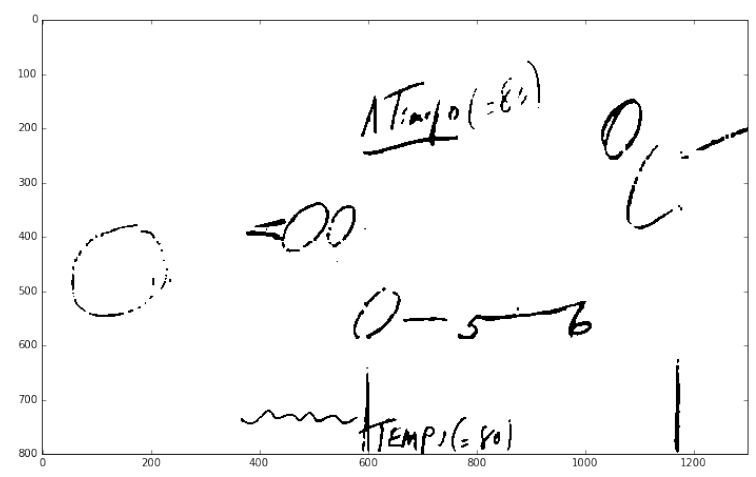

(d) Results of combined approach (Pipeline 3).

Fig. 4: Comparison of results of several heuristic annotation extraction pipelines. classifier was trained on the color values of a random subsample of the pixels in the original image array. The resulting image shows the locations of color-quantized pixels corresponding to the annotation color (blue), manually selected by inspecting the color cluster centers. Figure $4 \mathrm{c}$ shows the results of comparing marked and unmarked scores using Aruspix (Pipeline 2). Finally, Figure 4d shows the results of the combined approach, performed by training the classifier only on pixels that appear in the neighborhood of additions to the score, in order to improve the likelihood that the k-means algorithm converges on clusters that represent annotations (Pipeline 3). Additionally, the dilated mask revealing the neighborhood of annotations was reapplied before post-processing, eliminating mislabeled pixels distant from suspected annotation regions.

\subsection{Discussion}

While simple color separation (Pipeline 1) recovers parts of almost all of the original annotations, the results shown here indicate poor connectivity on large circular annotations and discontinuities in handwritten text. If the lighter parts of an annotation are sufficiently close to other non-annotation colors in the image, their color values may not be clustered with the color values of annotated regions of the image. The score image comparison pipeline (Pipeline 2) works well to address this issue, since any addition to the clean score will appear in the output of Aruspix's score comparison tool. However, the circular annotations are still not fully connected, interrupted by erasures corresponding to staff lines, which appear in both annotated and clean copies of the printed music.

Connectivity of large annotations is most improved in the results of the combined pipeline (Pipeline 3), though the legibility of handwritten text is arguably superior in the results of straightforward score image comparison. The combined approach has the potential to remedy some of the issues of annotation extraction by color separation. Image comparison remains promising in general and, in particular, for cases where no color scan of the annotated score is available or where dark gray/black annotations are preferred by the annotator. In particular, we believe the combined approach demonstrates a novel joint application of image comparison techniques to improve the results of an unsupervised method of annotation extraction. The output of the score comparsion pipeline can be converted to a mask, which is dilated and applied to the original image. Pixels revealed by the mask are used to train the unsupervised classifier to cluster pixels based on their percieved color similarity. This subset of the original image pixels that includes most of the annotated pixels. 


\section{Supervised annotation extraction}

\subsection{Background}

Recent work has shown that a battery of classical supervised machine learning algorithms perform well on the task of automatic staff-line removal in monochrome images [1]. Most generally, the supervised machine learning algorithms discussed there propose a function that maps from some realvalued feature space onto a set of binary class labels, which correspond to a judgement as to whether the current pixel is or is not part of a staff line.

We observe that this framework is adaptable to the task of handwritten annotation extraction. In our approach, each pixel in an annotated score image is associated with some point in the feature space. The goal is establish a function that can tell us if the pixel is likely to be part of a handwritten annotation (1) or the underlying printed score (0), based on features of the digitized score image that are local to the pixel in question. Supervised classification algorithms, such as those we present here, "learn" this function by ingesting a training set of feature-class pairs. After training a classifier in this way, the now-trained classifier can be used to generate judgments about score images which were not included as part of the training set.

The advantage of this approach is evident when we consider that while manual identification of handwritten annotations takes place in the order of minutes or hours, a welltrained classifier can identify annotations with reasonable recall in a matter of seconds. Our experience shows that these algorithms perform well on collections of score images that were annotated by the same annotator, and digitized under similar environmental conditions. With regard to annotation extraction from a heterogenous collection of score images, for example, consisting in an entire orchestral score marked up by the same annotator and photographed for archival purposes in the same session, we suggest that supervised machine learning techniques have the potential to reduce the workload required to extract handwritten annotations by a factor equivalent to the number of images in such a collection.

\subsection{Training and testing data}

The pixel-level features are the total collection of the color intensity values of pixels in an n-by-n-pixel window centered on the pixel being classified. Accordingly, there are $3 n^{2}$ features for each pixel, with the window size parameter $n$ allowed to vary in our experiments below. This choice is motivated by the intuition that annotations exhibit a local geometric structure that will be captured by a sufficiently large window size, to be determined experimentally.

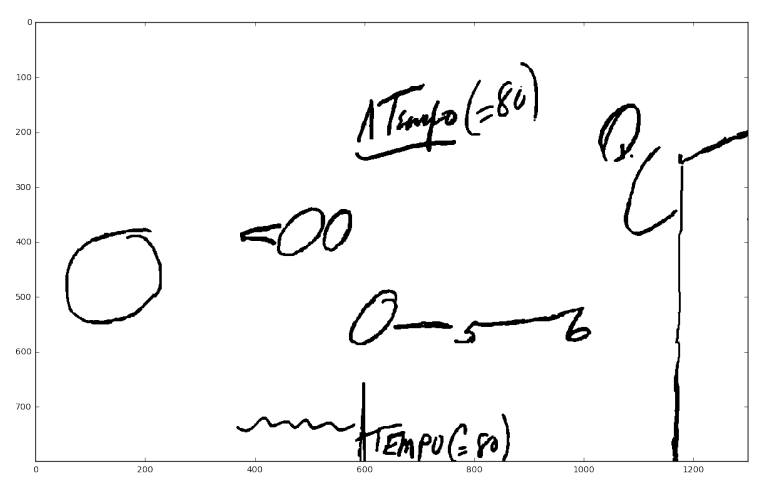

(a) Manually corrected class labels from output of Pipeline 3. Black pixels are annotations.

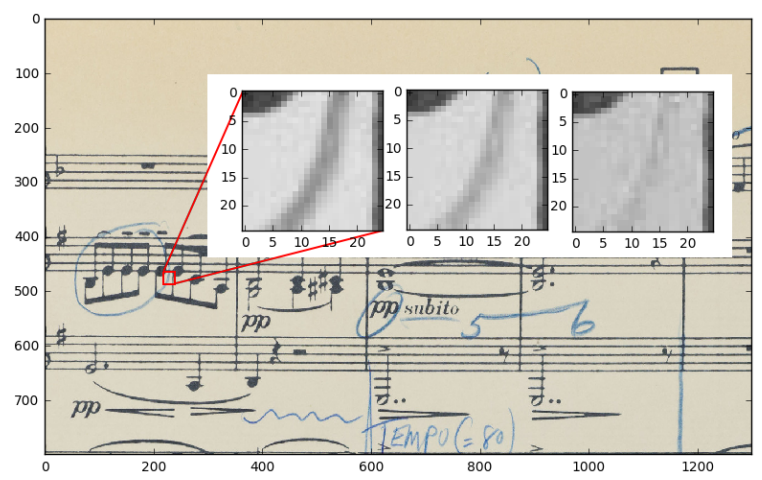

(b) Visualization of typical features used in supervised classifiers. Inset shows the pixel intensities in a 25x25-pixel neighborhood for the red, green, and blue channels, from left to right respectively.

Fig. 5: Visualization of manually curated class labels and feature space.

We can evaluate the performance of a proposed function by asking the function to make class-label predictions for pixels witheld from the algorithm during the training step, and comparing these predictions with class labels known in advance. To achieve this, we used hand-corrected results of the heuristic annotation extraction Pipeline 3 described above as our ground truth. We curated class labels for two annotated pages (PAGE3, PAGE27) chosen arbitrarily from the orchestral score of Mahler's Kindertotenlieder, annotated by Leonard Bernstein during his tenure at the New York Philharmonic.

We remark that the training data exhibit imbalanced classes. Only $2 \%$ of pixels in a representative feature-class pair were labeled as annotations. The performance of classifiers on imbalanced binary class data can be improved in certain cases by oversampling feature-class pairs from the minority class or undersampling from the majority class. We evaluate the effects of these techniques in our experiments below, focusing on the $F 1$ metric. 


\subsection{Experiments}

We ran three experiments designed to demonstrate the applicabilty of the supervised classification approach to handwritten score annotation.

First, we trained random forest classifiers on three families of training feature-class pairs derived from the PAGE3 ground truth: the imbalanced data; a synthetic dataset constructed by randomly undersampling the majority class for a 50:50 class balance; and another dataset constructed using Synthetic Minority Oversampling Method (SMOTE) to achieve a similar 50:50 class balance [2]. The contributed Python module imbalanced-learn was used to implement the resampling [7], while scikit-learn was used throughout to train the supervised learning algorithms. We computed the mean $F 1$ score of the classifier for each training set against the same test set $(n=10,000)$ taken from the unresampled data, to summarize the performance of trained classifiers as function of training set size, and to investigate the effect (if any) of rebalancing the dataset on the performance random forest classifiers in this task.

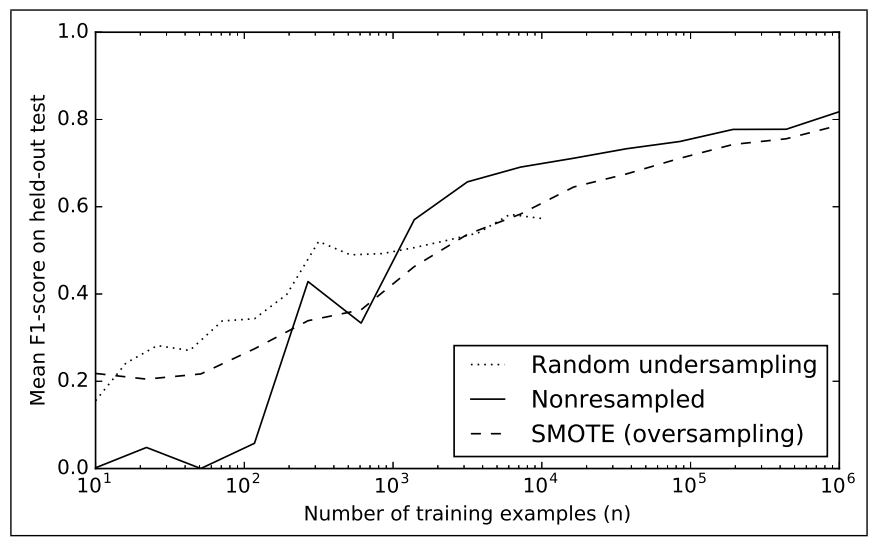

Fig. 6: Plot of mean F1 metric over batches of five runs of a random forest classifier trained on three datasets derived from PAGE3 ground truth, as a function of training set size.

In a second experiment, we evaluated the impact on classifier performance as the number of features is increased. In this experiment, we used two kinds of classifiers, a random forest classifier, and a k-nearest-neighbors classifier. The scikit-learn module for Python was used with the default hyperparameters to train and evaluate the classifiers. Only the window size (number of features) was allowed to vary. The performance metric reported in each case is the median F1 score using three-fold cross-validation. To compare the performance of random forest $(\mathrm{RaF})$ classifiers against k-nearest-neighbor ( $\mathrm{kNN}$ ) classifiers, a training subset of $100 \mathrm{k}$ feature-class pairs was used; to demonstrate the effective-

\begin{tabular}{lllllll}
\hline & & \multicolumn{4}{c}{ Window size (number of features) } \\
\cline { 3 - 7 } Classifier $(\mathrm{n})$ & Dataset & $1 \times 1$ & $3 \times 3$ & $5 \times 5$ & $7 \times 7$ & $9 \times 9$ \\
\hline $\mathrm{kNN}$ & PAGE3 & 72.2 & 78.5 & $\mathbf{8 2 . 4}$ & 81.3 & 80.1 \\
$(\mathrm{n} \approx 100 \mathrm{k})$ & PAGE27 & 71.1 & 81.3 & 82.7 & 82.0 & $\mathbf{8 3 . 1}$ \\
\hline $\mathrm{RaF}$ & PAGE3 & 68.5 & 76.5 & $\mathbf{8 0 . 3}$ & 78.7 & 80.0 \\
$(\mathrm{n} \approx 100 \mathrm{k})$ & PAGE27 & 68.5 & 79.1 & 78.3 & $\mathbf{8 1 . 0}$ & 79.1 \\
\hline \hline $\mathrm{RaF}$ & PAGE3 & 70.4 & 80.0 & 82.6 & 83.5 & $\mathbf{8 3 . 8}$ \\
$(\mathrm{n} \approx 1 \mathrm{M})$ & PAGE27 & 70.3 & 82.4 & 85.0 & $\mathbf{8 5 . 5}$ & 85.4 \\
\hline
\end{tabular}

Table 1: Comparison of classifier performance (median F1 score over three-fold cross-validation, expressed as a percentage) as a function of window size (number of features). $n$ indicates the number of training examples seen by the classifier in each training batch.

ness of random forest classifiers on larger training sets, a larger subset $(1 \mathrm{M})$ of training examples was used.

In the third and final experiment, we prepared a selection of class predictions made by a high-performing classifier (random forest, $7 \times 7$ feature windows, training set PAGE27), visualized by revealing the underlying original image pixel for each predicted annotation pixel, shown in Figure 7 and discussed below. These predictions demonstrate the ability of well-trained classifier to identify handwritten annotations in a variety of settings.

\subsection{Results}

Figure 6 shows that the performance of the classifier increases as a function of training set size. We note that the synthetic datasets (both the oversampled minority and undersampled majority) improve performance significantly when few training examples are provided, but this advantage is lost after about 1000 examples. Furthermore, though it is interesting to remark that classifiers trained on oversampled training data almost matches the performance on unresampled data in the limit of training set size, there was no benefit in computation time to prefering oversampled data in this case, and we used the original, unresampled training example data in the rest of our experiments.

The results of the second experiment show that, in general, an increase in window size (number of features) improves classifier performance, though an increase from a $7 \times 7$ window shape to $9 \times 9$ entails a marginal performance increase, and in some cases reduced the performance of classifiers. While the performance of k-nearest neighbor (kNN) classifiers is competitive with that of random forest classifiers given 100k training examples, class predictions by a kNN classifier are significantly slower in our implementation. As an illustrative example, 10k class predictions by a trained random forest classifier took $150 \mathrm{~ms}$, while predic- 


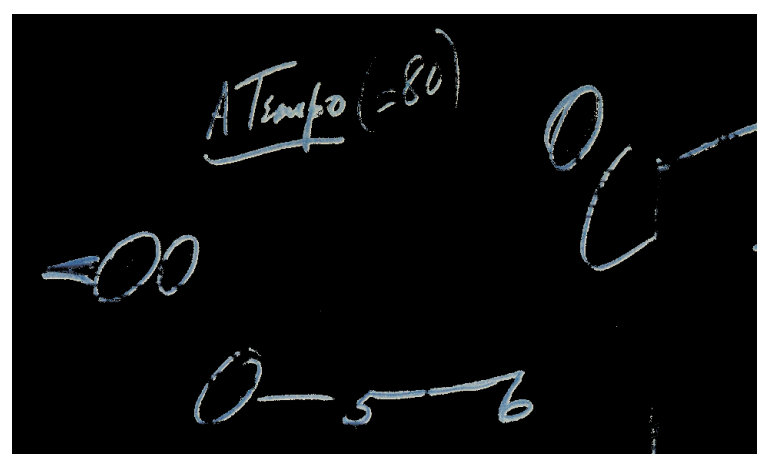

(a) From PAGE3 dataset. Mahler, Kindertotenlieder, p. 3. Marking artist: Leonard Bernstein.

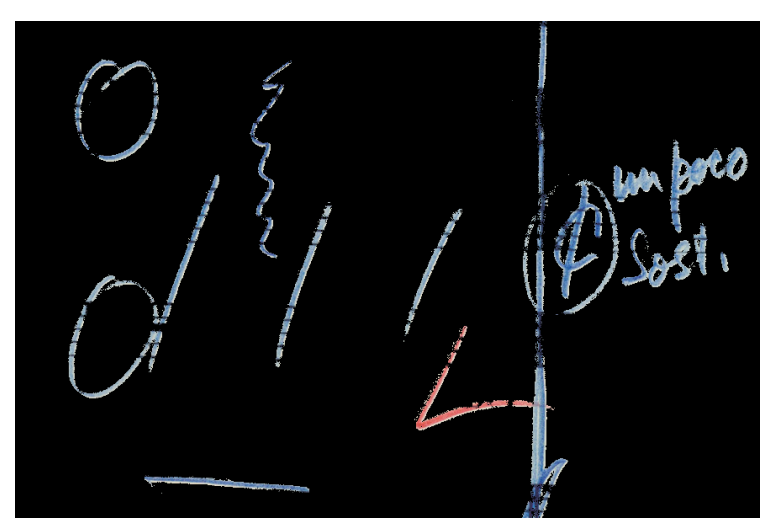

(b) From p. 34 of Mahler, Kindertotenlieder. Marking artist: Leonard Bernstein.

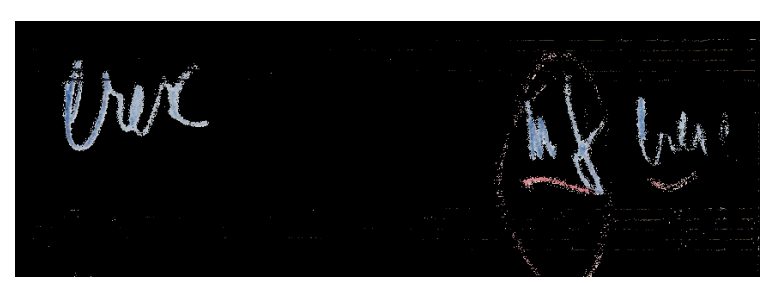

(c) From Beethoven, Overture to Coriolan. Marking artist: Leonard Bernstein.

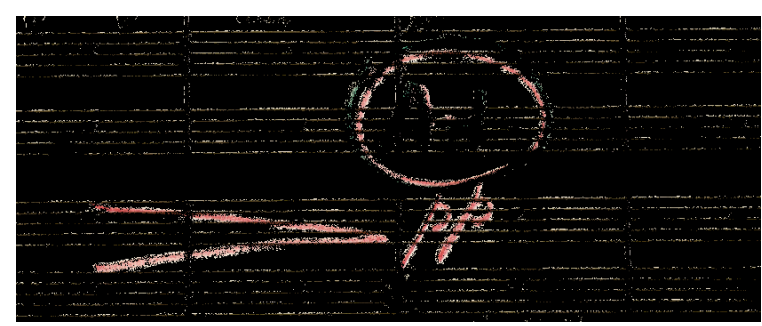

(d) Failure case. From Beethoven, Piano Concerto No. 5 ('Emperor'). Marking artist: Andre Kostelanetz.

Fig. 7: Visualization of class predictions from a random forest classifier trained on about $1 \mathrm{M}$ feature-class pairs from the curated PAGE27 data. tions by a kNN classifier took almost two orders of magnitude longer (14s) For this reason, an experiment validating a $\mathrm{kNN}$ classifier on $1 \mathrm{M}$ training examples was not performed. Our results show promise for random forest classifiers trained on about $1 \mathrm{M}$ training examples, using the feature space defined by the intensities of each color channel from pixels in a $7 \times 7$ window around the pixel to be classifed.

The third and final experiment reveals the qualitative successes and failures of our approach. Figure 7a shows a region of detected annotations from PAGE3. Figure $7 \mathrm{~b}$ shows a region of detected annotations from another page (p. 34) in the same copy of Kindertotenlieder marked by Bernstein. Recall that no training data was ingested for this page. Figure $7 \mathrm{c}$ shows a region from a different score (Beethoven, Coriolan Overture), again marked by Bernstein, which varies from the training examples in its color balance, particularly in the color of the page and printed ink. Finally, Figure 7d shows the results of predicting annotations on a page of a score marked up by a different artist (Andre Kostelanetz) from a different batch of score scans. These final two prediction sets are offered as failure cases, showing that the success of this method in predicting annotations within a single source, which is typically digitally imaged under similar lighting and color-balance conditions, is dependent both on the environmental conditions unique to the digitizing process and on the author's annotation habits.

\subsection{Discussion}

Each batch of classifiers was used with the default parameters provided "out of the box" by scikit-learn. Our goal was not to present the ideal tuning for these supervised approaches, nor to suggest that these algorithms are optimal for the task at hand. Rather, we suggest that these indicators of the success of a naive application of supervised classification algorithms to the problem of score annotation extraction points to a fruitful line of further research. In Section 4, we discuss how the development of a curated dataset of labeled score images that is representative of a diversity of handwriting styles, score styles, and digitization environments, will provide ground truth that will be used to develop and evaluate handwritten annotation extraction methods that are more robust.

\section{Applications and prospects}

\subsection{Applications in digital libraries}

A wide range of applications of interest to digital libraries with digitized musical score holdings can be imagined that are based on the results of the techniques described above. 
Extracted annotations can be converted to vector graphics for integration into an encoded version of the score. The latest version of the widely-supported Music Encoding Initiative XML schema supports the integration of SVG shapes within encoded music scores, using the <graphic> element.

This would make it possible for users to interactively and dynamically visualize different annotation sets that were made for the same underlying score edition, enabling interactive critical web editions of performance scores. Encoded scores enriched in this way could lead to the use of annotations in the preparation of real or virtual performances that are musically informed by the performance decisions preserved in a diverse corpus of conductor annotations.

Dedicated visualization environments for the systematic investigation of score annotations can also be imagined. For example, extracted annotations could be grouped by shape using existing geometric classification techniques. In combination with encoded score data, this would enable a user to browse and filter annotations by their score location, their associated metadata (such as year of annotation), or their shape.

Several scores exist that have been annotated by more than one conductor, sometimes with a single score being annotated by several conductors. Extracting the annotations is a first step towards eventually understanding the distinctive annotation practices of specific conductors, and towards testing authorship attribution hypotheses based on the content and structure of known-author score annotations.

\subsection{Prospects}

Though the extraction processes described here produce promis ing results a more automated workflow for score annotation extraction requires further work. For instance, during the score comparison step, Aruspix discards image color channel information that is useful to annotation extraction. One possible improvement would be to modify the Aruspix algorithm in order to preserve this information and to make subsequent use of it throughout the comparison pipeline. Also, at present, an affine transformation must be applied to Aruspix's "image diff" in order to match the geometry of the original score image for use in the masking step of the combined heuristic pipeline, though this issue concerns the interface to Aruspix, and not the underlying algorithms it utilizes. Improvements in the case of discontinuites due to staff lines can be achieved by applying a staff removal algorithm to the annotated score image prior to extraction, such as those evaluated in [3]. It is expected that this would improve the connectivity of annotations that overlap staff lines.

In the present approach, unmarked copies of score images are used only in the heuristic approaches to annotation extraction, as the basis for image comparison. A suitably aligned and pre-processed unmarked copy could be used to generate an additional set of class labels so that the pixel classification task could be extended beyond simple binary classification. Other improvements are readily suggested by the vast literature on machine learning applications in computer vision, including those areas related to feature selection, scalability, and the recent groundswell in interest in neural networks to perform scene segmentation on images [10].

Once the annotations can be reliably extracted from a score image, they must be segmented systematically associated with the underlying score symbols in order to make sense of their meaning and function, which often depends on the musical context provided by the score. Robust rules for associating annotations to score symbols remain to be developed.

Any future research in handwritten annotation extraction in this domain would greatly benefit from a curated dataset of score images and their associated annotation labels. This dataset would facilitate novel model development and tuning under the test-train-validate regime of model improvement. It should go without saying that such a dataset must be representative of a wide variety of annotation styles, annotation media (pens, crayons, and pencils), score types, and score digitization methods.

This ground truth could well be crowdsourced, with candidate annotation regions being determined using the output of heuristic annotation extraction methods described above. Users can be asked to trace over the annotations in a score image fragment, and such an interface could be gamified with the help of heuristic extraction methods with which a scoring function may be implemented that feeds back an estimate of the user's performance as they complete the labeling task. A similar user recruitment strategy is outlined in [8], where gamification was used to motivate user to provide line tracings of portrait photography, for later use in a probabilistic model of photograph tracing. This kind of interface would have the beneficial side-effect of driving engagement with these novel archival holdings, particularly by users beyond beyond the typical institutional consumer of online archival content. Alternatively, expert annotators equipped with digitizing tablets and off-the-shelf photo editing software (such as Adobe Photoshop or GIMP) could correct the output of a heuristic extraction pipeline, as we have done in the present paper.

\section{Conclusions}

The increasing number of musical score image digitization projects is making evidence of historical and institutional performance practice more widely available than ever before. This evidence is captured in the handwritten annotation practices of performers that fill the pages of conductors' 
copies of orchestral scores. The fully automated extaction of these annotations remains an open problem for the music information retrieval and document analysis communities. However, by identifying some of the domain-specific affordances of conductors' score annotation practices-including the use of colored annotations and the ready availability of thousands of unmarked score images online-we have made a contribution to this area of interest.

We have proposed an number of heuristic image processing pipelines that perform annotation extraction relatively robustly using simple methods. Then, we demonstrated the advantages of adopting a machine learning approach to annotation extraction, by framing the task as a supervised classification problem and running a number of experiments on a small test set of feature-class label pairs. Of course, supervised learning methods require labeled data for training, and we showed how our heuristic annotation extraction techniques expedite the curation of expert-annotated ground truth data. We propose the preparation of a much larger, representative corpus of score images and their associated annotation class labels to facilitate further development of annotation extraction algorithms in this domain. With a reliable algorithm for score annotation extraction in hand, only then may we go on to develop rich interactive interfaces with the growing digital archive of musical scores, and motivate the systematic research of score annotation and their associated musical and interpretative practices.

Acknowledgements The first author wishes to thank Barbara Haws at the New York Philharmonic Archives and Mitchell Brodsky for their technical support and encouragement. Original score image courtesy Leon Levy Digital Archive, New York Philharmonic.

\section{References}

1. Calvo-Zaragoza J, Mic L, Oncina J (2016) Music staff removal with supervised pixel classification. International Journal on Document Analysis and Recognition (IJDAR) 19(3):211219, DOI 10.1007/s10032-016-0266-2, URL http://link.springer.com/article/10.1007/s10032-0160266-2

2. Chawla NV, Bowyer KW, Hall LO, Kegelmeyer WP (2002) Smote: synthetic minority over-sampling technique. Journal of artificial intelligence research 16:321357

3. Dalitz C, Droettboom M, Pranzas B, Fujinaga I (2008) A comparative study of staff removal algorithms. IEEE Transactions on Pattern Analysis and Machine Intelligence 30(5):735-766

4. Fan KC, Wang LS, Tu YT (1998) Classification of machine-printed and handwritten texts using character block layout variance. Pattern Recognition 31(9):12751284

5. Farooq F, Sridharan K, Govindaraju V (2006) Identifying handwritten text in mixed documents. In: 18th International Conference on Pattern Recognition (ICPR'06), vol 2, pp 1142-1145, DOI 10.1109/ICPR.2006.676

6. Guo JK, Ma MY (2001) Separating handwritten material from machine printed text using hidden Markov models. In: Proceedings of the Sixth International Conference on Document Analysis and Recognition, pp 439-443, DOI 10.1109/ICDAR.2001.953828

7. Lemaître G, Nogueira F, Aridas CK (2016) Imbalancedlearn: A python toolbox to tackle the curse of imbalanced datasets in machine learning. CoRR abs/1609.06570, URL http://arxiv.org/abs/1609.06570

8. Limpaecher A, Feltman N, Treuille A, Cohen M (2013) Real-time drawing assistance through crowdsourcing. ACM Transactions on Graphics 32(4):1, DOI 10.1145/2461912.2462016, URL http://dl.acm.org/citation.cfm?doid=2461912.2462016

9. Nakai T, Kise K, Iwamura M (2007) A method of annotation extraction from paper documents using alignment based on local arrangements of feature points. In: Proceedings of the Ninth International Conference on Document Analysis and Recognition, IEEE, vol 1, pp 23-27

10. Papandreou G, Chen LC, Murphy K, Yuille AL (2015) Weakly-and semi-supervised learning of a DCNN for semantic image segmentation. arXiv preprint URL http://arxiv.org/abs/1502.02734

11. Peng X, Setlur S, Govindaraju V, Sitaram R (2011) Handwritten text separation from annotated machine printed documents using Markov Random Fields. International Journal on Document Analysis and Recognition (IJDAR) 16(1):1-16, DOI 10.1007/s10032-0110179-z

12. Pugin L (2006) Aruspix: an automatic sourcecomparison system. In: Hewlett WB, Selfridge-Field E (eds) Music Analysis East and West, Computing in $\mathrm{Mu}-$ sicology, vol 14, MIT Press, Cambridge, MA, pp 49-60

13. Violante S, Smith R, Reiss M (1995) A computationally efficient technique for discriminating between handwritten and printed text. In: IEE Colloquium on Document Image Processing and Multimedia Environments, IET, pp 17-1

14. Zagoris K, Pratikakis I, Antonacopoulos A, Gatos B, Papamarkos N (2014) Distinction between handwritten and machine-printed text based on the bag of visual words model. Pattern Recognition 47(3):1051-1062, DOI 10.1016/j.patcog.2013.09.005 